\title{
Suzaku View of Magnetic Cataclysmic Variables
}

\author{
T. Yuasa ${ }^{1}$ \\ ${ }^{1}$ Institute of Space and Astronautical Science (ISAS), Japan Aerospace Exploration Agency (JAXA), 3-1-1 Yoshinodai, \\ Chuo, Sagamihara, Kanagawa 252-5210, Japan
}

Corresponding author: yuasa@astro.isas.jaxa.jp

\begin{abstract}
Results of magnetic cataclysmic variable studies performed with the Suzaku satellite are reviewed in this article. Particular emphasis is placed on the recent update of X-ray spectral model of intermediate polars, possible kinematically redshifted fluorescent Fe K emission lines, and the magnetic CV contribution to the Galactic ridge X-ray emission.
\end{abstract}

Keywords: cataclysmic variables - intermediate polars - X-rays.

\section{Introduction}

Magnetic cataclysmic variables emit hard X-rays from hot plasma with temperatures of $10^{8-9} \mathrm{~K}$ contained in the post-shock accretion column on the magnetic pole(s) of a white dwarf. Japan's X-ray observatory satellite Suzaku, providing X-ray CCD coverage below $10 \mathrm{keV}$ and hard X-ray response up to $50 \mathrm{keV}$ with the Si-PIN diode, has been actively observing magnetic CVs, particularly, intermediate polars through its initial observation phase to following AO phases. As of 2013, more than 20 intermediate polars have been observed, and main results have been reported in [11], [15], [13], and [3]. In the following sections, we review major results reported in these articles.

\section{X-Ray Spectral Model of Intermediate Polars}

In [15], we have constructed, based on the previous study by [10], an X-ray spectral model of the postshock plasma of intermediate polars, by numerically solving the three conservation equations (mass, momentum, and energy) with a newly reported plasma cooling function [9]. Since the shock temperature depends on the gravitational potential depth, this model contains a $M_{\mathrm{WD}} / R_{\mathrm{WD}}$ ratio as a free parameter, and by combining the $M_{\mathrm{WD}} / R_{\mathrm{WD}}$ ratio with a theoretical $M_{\mathrm{WD}}-R_{\mathrm{WD}}$ ratio (e.g. by [5]), one can estimate $M_{\mathrm{WD}}$ and $R_{\mathrm{WD}}$ from observed spectra.

Figure 1 shows an example of spectral fitting with the multi-temperature model. A single-temperature model apparently fails to reproduce the observed spectrum, especially in the $\mathrm{Fe} \mathrm{K}$ emission line region (1 upper panels). This can be understood as follows; the hard $\mathrm{X}$-ray continuum requires hotter temperature (due to emission from hot plasma close to the shock) although the apparent He-like and H-like $\mathrm{Fe} \mathrm{K} \alpha$ emission line ratios require much cooler plasma temperatures (the model underestimates the He-like $\mathrm{Fe} \mathrm{K} \alpha$ line and overestimate the $\mathrm{H}$-like $\mathrm{Fe} \mathrm{K} \alpha$ line).

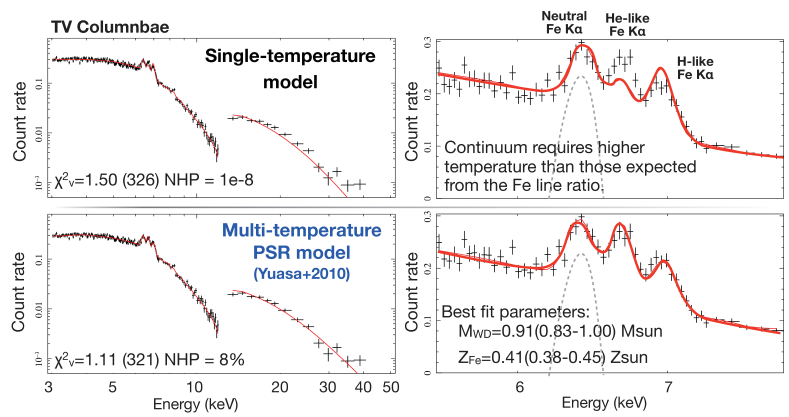

Figure 1: Suzaku spectra of TV Col fitted with a single-temperature model (upper panels) and our model [15] with emissivity gradient (lower panels). Crosses and red curves are observed source count rates and response-convolved model count rates. Right panels present blow-up view of the Fe emission line region. Note that in both cases, $6.4 \mathrm{keV}$ Fe K fluorescent line was modeled with a single gaussian of which normalization was allowed to freely vary.

Our multi-temperature model, on the other hand, successfully reproduces the Fe $\mathrm{K}$ emission lines as well as the over-all spectral shape. The best fit parameters give acceptable fit statistics, and a WD mass estimate of $0.91(0.83-1.00) M_{\odot}$. Figure 2 summarizes estimated WD masses of 17 IPs compared with the average WD masses of isolated and non-magnetic accreting systems. 


\section{$2.1 \quad$ Recent updates}

[2] has recently reported an updated version of the intermediate polar spectral model that takes into account several aspects in the model geometry and the numerical calculation; (i) the new model can have a dipolar accretion column geometry as well as a cylindrical column, (ii) conservation equations are set for ions and electrons separately, and (iii) the accretion rate can be variable. Figure 3 illustrates the accretion geometry which was considered in their paper. The two-fluid treatment is to account for the delayed increase of the electron temperature after the shock where ions are first heated, and electrons are collisionally heated relatively slowly.

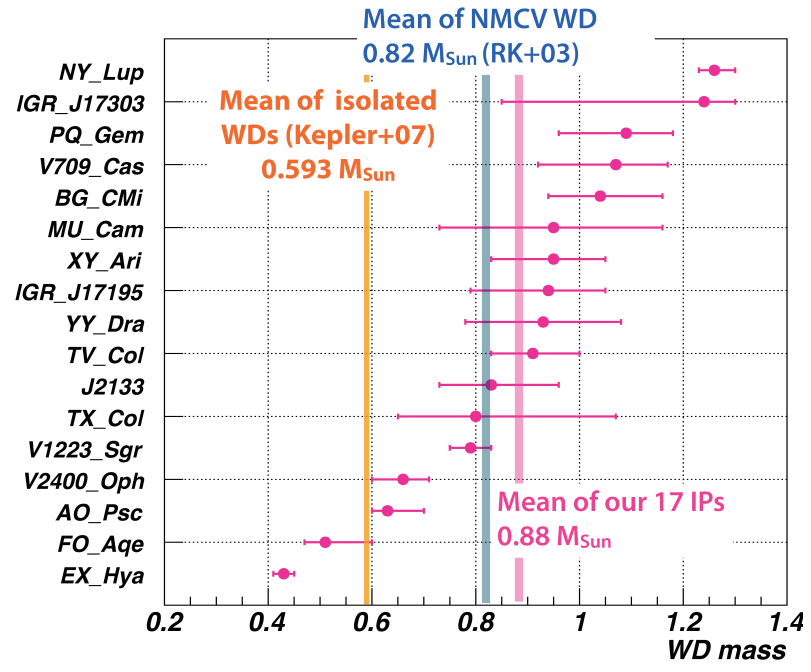

Figure 2: Distribution of estimated masses of WDs in 17 nearby intermediate polars [15]. Average WD masses are shown for isolated WDs [4] and non-magnetic CVs [8].

Broad-band spectra calculated by [2] are shown in Figure 4 together with ratios between different specific accretion rates at the shock. Major differences are expected, in particular, in lower accretion rates with dipolar geometry. Based on this, the authors expect that the estimated WD mass could have been artificially reduced in systems like EX Hya which has a low accretion rate.

\section{Spin Modulation of Centroid Energy of Fluorescent Fe $\mathrm{K} \alpha$ Emission Line}

[3] analyzed Suzaku data of V1223 Sgr and found a spin modulation of the centroid energy of the fluorescent Fe $\mathrm{K} \alpha$ emission line as shown in the third panel of Figure 5. The maximum energy shift in terms of centroid energy of a single gaussian was $\sim 20 \mathrm{eV}$. When the fluorescent line is fitted with two gaussians, one at $6400 \mathrm{eV}$ and the other having freely varying centroid energy, the minimum of the centroid energy of the second component is $6.29_{0.11}^{+0.10} \mathrm{keV}$.

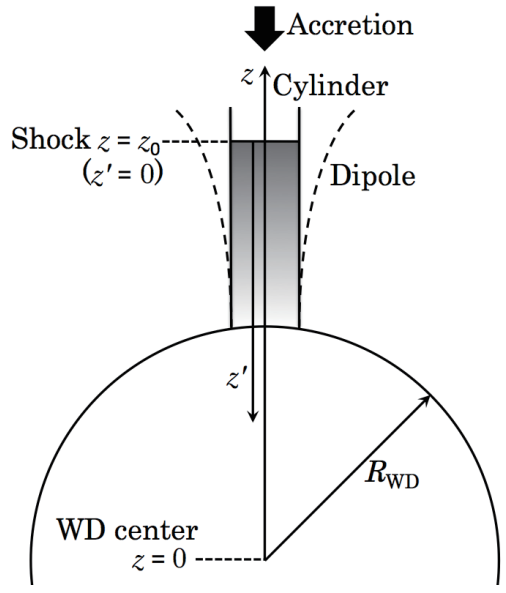

Figure 3: Dipolar and cylindrical geometry of the post-shock accretion region that was assumed in [2].
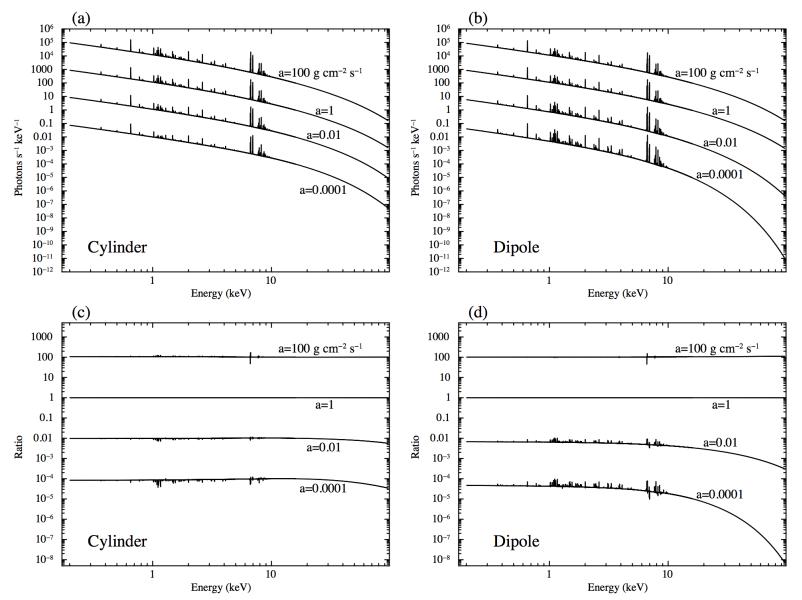

Figure 4: Upper panels: Model spectra calculated for $M_{\mathrm{WD}}=0.7 M_{\odot}$ with various specific accretion rates $a$ and (a) cylindrical and (b) dipolar accretion geometry. Lower panels: Ratio spectra normalized by that for $a=100 \mathrm{~g} \mathrm{~cm}^{-2} \mathrm{~s}^{-1}$.

The authors attributed this red-ward shift to the kinematic Doppler shift caused by reflection from the rapidly falling pre-shock accretion matter $\left(v_{\mathrm{ff}} \sim \mathrm{a}\right.$ few $\times 1000 \mathrm{~km} \mathrm{~s}^{-1}$ ), and interpreted that the spin modulation is caused by the changing viewing angle (timedependent radial velocity of the accreting pre-shock material). Although the detection of the second (redshifted) component is marginal in Suzaku data, the near future $A S T R O-H$ observatory will definitively detect this with splendid energy resolution of $\sim 5 \mathrm{eV}$ 
(FWHM), and will provide a new technique to estimate the gravitational potential depth of a WD from the magnitude of the energy shift.

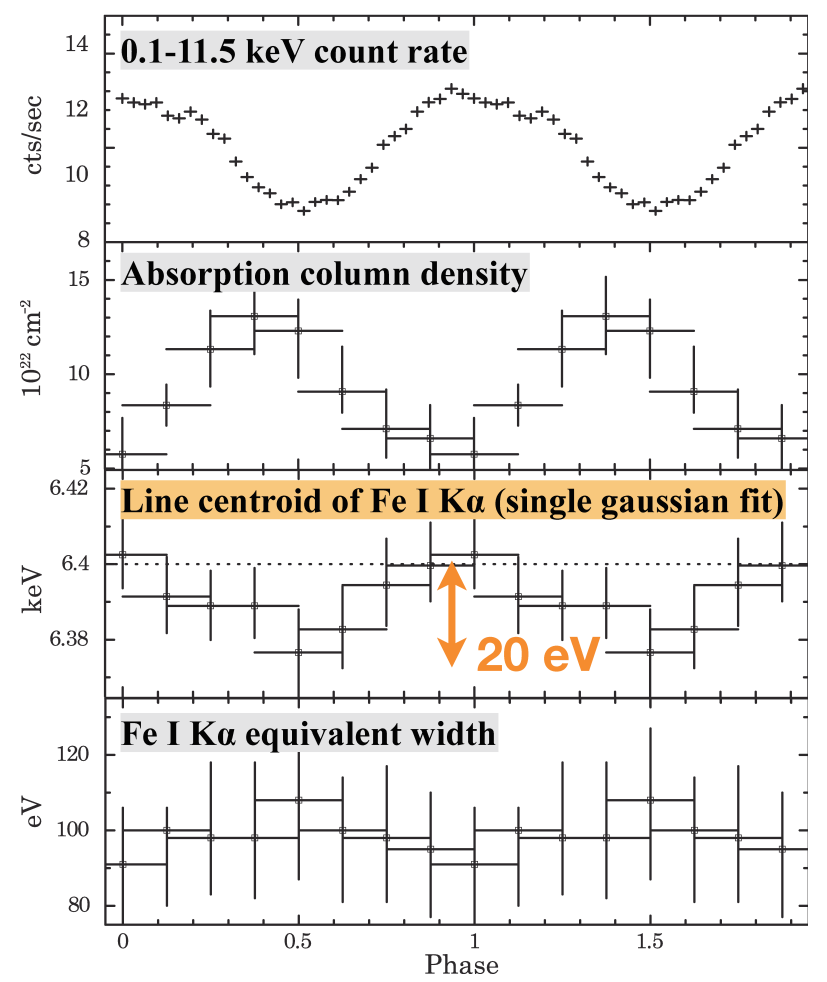

Figure 5: Spin-modulation of count rates, and best-fit parameters of absorption column density, centroid energy of fluorescent $\mathrm{Fe} \mathrm{K} \alpha$ emission line, and its equivalent width. Adopted from [3] with additional labels.

\section{Serendipitous Discoveries}

Although Suzaku X-ray telescope has a tightly collimated field of view of $\sim 18$ arcmin $\times 18$ arcmin, several new magnetic CVs are identified with their intense three Fe $\mathrm{K} \alpha$ emission lines as presented in Figure 6.

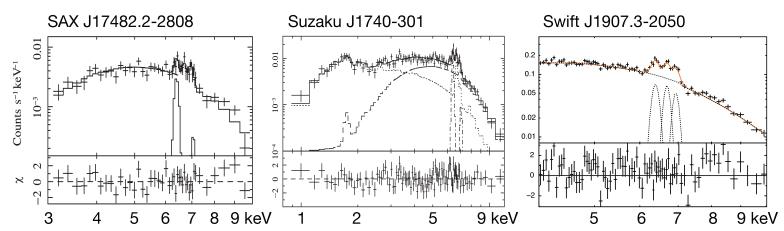

Figure 6: Suzaku/XIS spectra of serendipitous sources that are thought to be magnetic CVs. Adopted from [6], [12], and [1].

As claimed and spectroscopically confirmed in [7] and [14], respectively, magnetic CVs (more precisely intermediate polars) are the major contributors of the Galactic ridge X-ray emission (GRXE), so there should be enormous number of magnetic CVs in the field. These findings partially support this idea of omnipresence of hard X-ray emitting magnetic CVs in the Galaxy. Figure 7 shows a fit result of the broad-band GRXE spectrum with the IP model and an additional lower temperature component (which is attributed to superposition of a number of dim coronal sources).

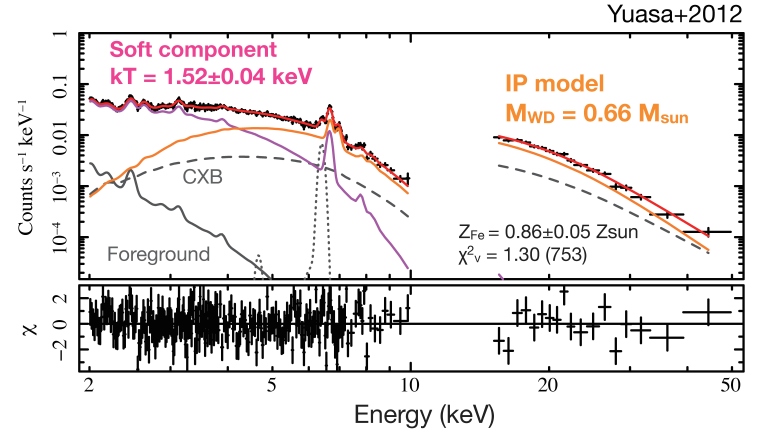

Figure 7: Broad-band spectral fitting of the GRXE using the intermediate polar spectral model [14].

\section{Conclusion}

Suzaku will continue to observe several magnetic CVs, for example, in 2013 (AO-8), V1223 Sgr will be reobserved for $180 \mathrm{ks}$ to reconfirm the spin modulation of the centroid energy of the fluorescent $\mathrm{Fe} \mathrm{K} \alpha$ emission line. The forthcoming ASTRO-H mission will provide a great step forward in understanding the accretion geometry, post-shock region, reflection, and many other physical processes taking place in magnetic CVs.

\section{References}

[1] Bernardini, F. et al., 2012, A\&A, 542, A22

[2] Hayashi, T., \& Ishida, M. 2013, ArXiv e-prints

[3] Hayashi et al., 2011, PASJ, 63, 739

[4] Kepler, S. O. et al., 2007, MNRAS, 375, 1315 doi:10.1111/j.1365-2966.2006.11388.x

[5] Nauenberg, M. 1972, ApJ, 175, 417 doi:10.1086/151568

[6] Nobukawa, M., Koyama, K., Matsumoto, H., \& Tsuru, T. G. 2009, PASJ, 61, 93

[7] Revnivtsev, M., Sazonov, S., Gilfanov, M., Churazov, E., \& Sunyaev, R. 2006, A\&A, 452, 169

[8] Ritter, H., \& Kolb, U. 2003, A\&A, 404, 301 
[9] Schure, K. M., Kosenko, D., Kaastra, J. S., Keppens, R., \& Vink, J. 2009, A\&A, 508, 751

[10] Suleimanov, V., Revnivtsev, M., \& Ritter, H. 2005, A\&A, 435, 191

[11] Terada, Y. et al. 2008, PASJ, 60, 387

[12] Uchiyama, H. et al., 2011, PASJ, 63, 865

[13] Yuasa, T. 2013, Suzaku Studies of White Dwarf Stars and the Galactic X-ray Background Emission, Springer Theses Ser. (Springer)

[14] Yuasa, T., Makishima, K., \& Nakazawa, K. 2012, ApJ, 753, 129 doi:10.1088/0004-637X/753/2/129

[15] Yuasa, T. et al., 2010, A\&A, 520, A25

\section{DISCUSSION}

S. BALMAN: Have you checked any correlation between $M_{\odot}$, temperature, and luminosity in your paper on the Suzaku sample?

T. YUASA: There was no correlation for any parameter such as $M_{\odot}$, temperature, or luminosity.

S. SCARINGI: Does the geometry of the post-shock region affect the WD mass estimation?

T. YUASA: Yes, it affects it as explained in [2]. Quantitatively, the dipolar case poses geometrical compression which leads to increased emissivity and therefore lower apparent plasma temperature compared to the cylindrical case. 